\title{
The research of the parameters of a vibration machine for composite materials compaction
}

\author{
Alexander Maslov, ${ }^{1,}$, and Janar Batsaikhan ${ }^{2}$ \\ ${ }^{1}$ Kremenchuk Mykhailo Ostrohradskyi National University, Ukraine \\ ${ }^{2}$ Research and production center «MCPCgr», Mongolia
}

\begin{abstract}
Based on the analysis of the conventional methods for composite materials compaction, the authors propose a vibration machine made as a form without bottom with a horizontal oscillation vibro-exciter mounted on its end face. To determine the rational parameters of the vibration machine we researched the "vibration form - composite medium" dynamic system wherein composite medium is represented in the form of a system with distributed parameters. This system takes into account the resilient, viscous, inertia and power properties of the compacted composite material. We formulated a partial differential equation describing the variation of stresses in the compacted medium depending on the dynamic module of resilient deformation, the coefficient of dynamic viscosity, the coefficient of non-resilient resistance and the compacted medium inertia in the functional dependence on the composite material density and relative deformation. We formulated an oscillation wave equation describing the propagation of the viscous-resilient-plastic waves of deformation in the compacted composite material. The solution of the oscillation wave equation resulted in the formulation of the law of the oscillation of the form and the compacted material. We determined the stresses occurring in the composite material. We obtained the relations for the determination of the vibration machine basic parameters depending on the physical and mathematical characteristics of the compacted composite material.
\end{abstract}

\section{Introduction}

Polymeric composite materials are more popular in comparison with others due to the simplicity of production, adaptability to manufacture and low price. We should mark out a vibration process of compaction among the conventional processes of composite material shaping $[1,2,3]$ due to its low power consumption and high efficiency. However, in spite of the listed advantages, vibration pressing is comparatively seldom used in industry. It results from the limited possibilities of this method in formation of big-size parts and also from insufficient study of the vibration machine interaction with the compacted material. For the efficient operation of the vibration machine it is necessary to accurately select its basic parameters and the modes of vibratory action on the compacted medium represented

\footnotetext{
*Corresponding author: kmto.43@gmail.com
} 
by a rheological model in the form of a Hooke's resilient model, Newton's viscous body, resilient-viscous body of Kelvin-Vogt of Maxwell [4 - 5]. It is possible to obtain sufficiently accurate results if the compacted medium is represented in the form of continuum [ $6-8]$, and its oscillations are described by a wave equation [9], taking into account the resilient and viscous properties of the compacted material. When these methods are used, the inertia properties and friction forces of the composite material components are not taken into consideration at their reorientation, approaching, deformation. That is why to choose the basic parameters of the vibration machine and the modes of vibratory action on the compacted material it is necessary to use a rheological model for the continuum with distributed parameters, which takes into account the resilient and non-resilient resistive forces as well as resistances caused by the action of the internal friction forces and the inertia of the composite material.

The purpose of the present research consists in the determination of the vibration machine rational parameters based on the analysis of its interaction with the compacted composite material represented by a system with distributed parameters.

\section{The material and results of the research}

The physical and mathematical properties of the compacted medium influence greatly the character of the oscillation of the vibration machine used for compacting composite materials. It is the correct account of all the motive and resistive forces that makes it possible to determine the regularity of the vibration process of composite materials compacting with a sufficient degree of accuracy, to identify the rational parameters of the vibration machine whose design is shown in Fig. 1. The vibration machine contains a bottomless form consisting of longitudinal 1 and transversal 2 boards. In the upper and lower parts of the form there is a binding made of metal L-bars 3 and 4 . Bracket 5 is rigidly mounted on the form end face; horizontal oscillation vibratory exciter 6 is mounted on the bracket bed-plate. L-bars 4, forming the lower binding are equipped with sealing rubber elements 7 . The lower part of bracket 5 bears support plate 8 , equipped with damper 9 . The vibration machine is freely installed on flat surface 10 .

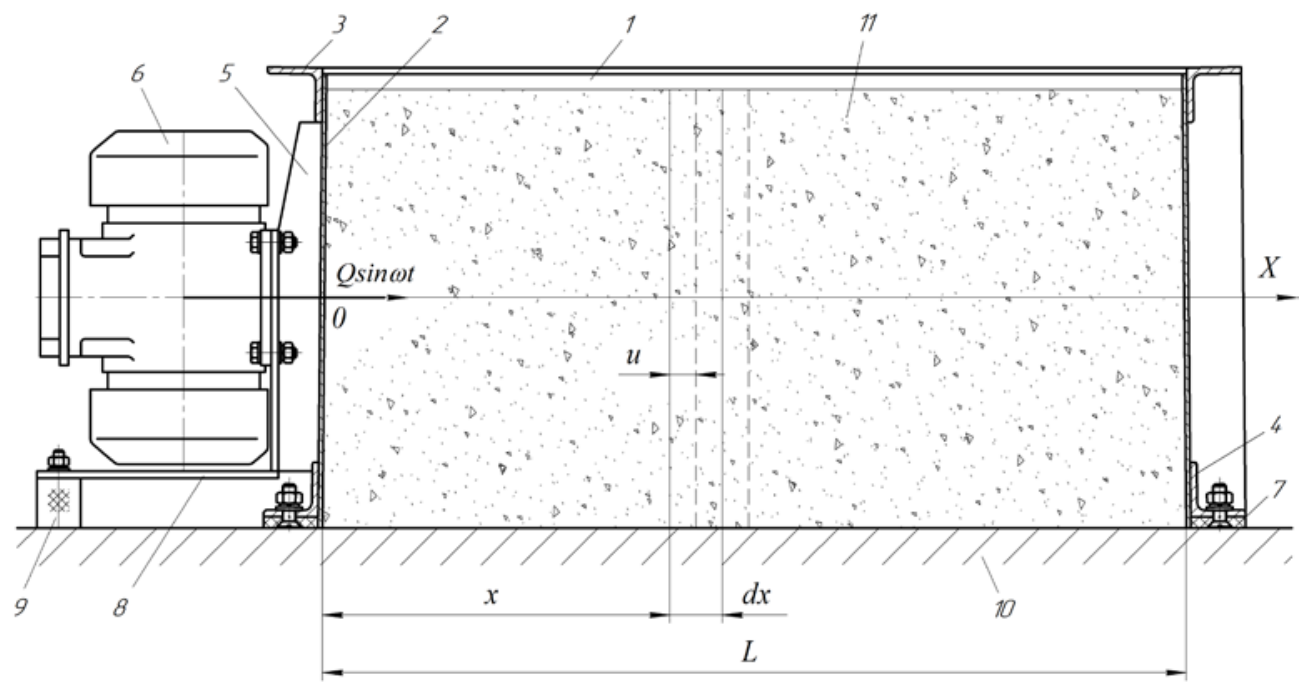

Fig. 1. The vibration machine design diagram.

The vibration machine operates in the following way. The form is filled with composite 
material 11 and oscillation vibratory exciter turns on, the form performs horizontal oscillations under its action, and the composite material undergoes complex stressdeformed action resulting in the destruction of internal links, decrease of the internal friction and creation of a more compact packing. After the completion of the formation process the vibration machine moves vertically upwards, separates from the formed composite and shifts to a new operation position.

To analyze the law of the motion of the vibration machine (Fig. 1), we represent the compacted medium in the form of a rheological model (Fig.2), taking into account the action of the resilient, dissipative and inertial forces occurring in this medium under dynamic action.

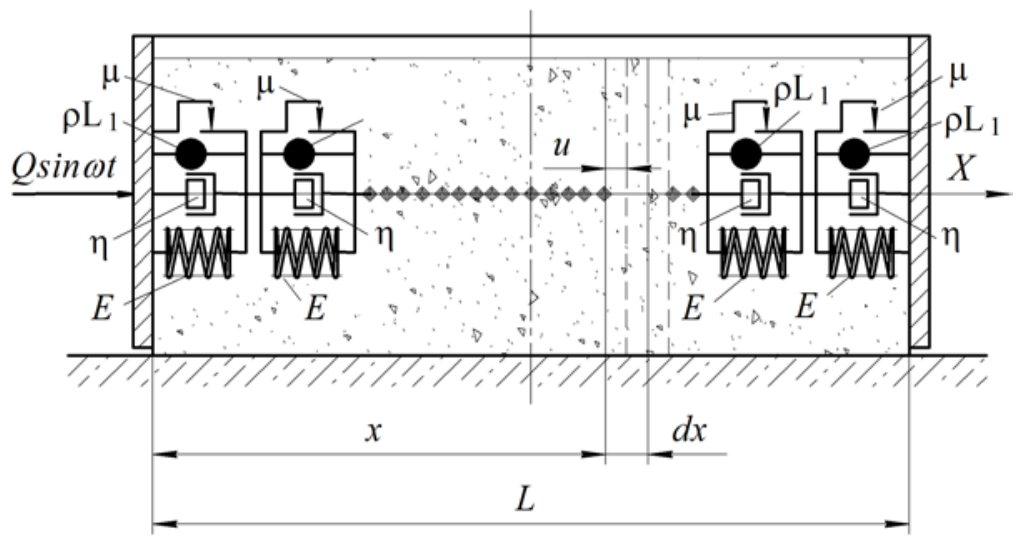

Fig. 2. A rheological model of the compacted medium.

In accordance with the adopted rheological model, we research uniaxial stressed state occurring in the compacted medium between the face walls of the form under the action of vibratory excitation $Q \sin \omega t$. We represent the dependence between the stress and deformation in the compacted medium in the form of an equation taking into account the action of resilient, dissipative and inertial forces essentially influencing the stress occurrence:

$$
\sigma(x, t)=E \frac{\partial u(x, t)}{\partial x}+\eta \frac{\partial u(x, t)}{\partial t}-\rho L_{1} \frac{\partial^{2} u(x, t)}{\partial t^{2}}+\mu u(x, t),
$$

where $\sigma(x, t)$ - stresses occurring in the compacted layer in the direction of the coordinate axis $X ; u$ and $x$-Eulerian and Lagrangian coordinates; $E$ - the dynamic module of the composite material resilient deformation; $\eta$-the dynamic viscosity coefficient taking into account the internal friction in the compacted layer; $\rho$ - the composite material density; $L_{1}$ - the reduced effective thickness of the compacted layer in the direction of the coordinate axis $X ; \mu$ - resistance coefficient taking into account the amount of energy required to destroy the internal links, air displacement, particles reorientation and other phenomena in the compacted medium, which accompany the vibration compaction [9].

The compacted composite layer oscillations in the direction of coordinate $X$ in time $t$ will be represented by a known dependence [9]:

$$
\frac{\partial \sigma(x, t)}{\partial x}=\rho \frac{\partial^{2} u(x, t)}{\partial t^{2}},
$$

which, based on expression (1), results in the following equation of wave oscillations - 


$$
E \frac{\partial^{2} u(x, t)}{\partial x^{2}}+\eta \frac{\partial^{2} u(x, t)}{\partial x \partial t}-\rho L_{1} \frac{\partial^{3} u(x, t)}{\partial x \partial t^{2}}+\mu u(x, t)=\rho \frac{\partial^{2} u(x, t)}{\partial t^{2}} .
$$

The solution to equation (3) can be represented in the form of the imaginary part of the complex function [9]:

$$
u(x, t)=U(x) e^{i \omega t},
$$

where $U(x)$ - oscillations complex amplitude that is to meet the edge (boundary) conditions for the design diagram shown in Fig. 1.

We will look for the solution to the presented oscillation equation (3) at the following boundary conditions:

$$
\begin{aligned}
-m \frac{\partial^{2} u(0, t)}{\partial t^{2}}-b \frac{\partial u(0, t)}{\partial t}-c u(0, t)+E F \frac{\partial u(0, t)}{\partial x}+\eta F \frac{\partial u(0, t)}{\partial t}- & \\
-\rho L_{1} F \frac{\partial^{2} u(0, t)}{\partial t^{2}}+\mu F u(0, t) & =Q \sin \omega t \\
u(L, t) & =u(0, t)
\end{aligned}
$$

where $m$ - the mass of the vibration machine; $c$ - the reduced rigidity of the damper and the rubber seals; $F$ - the area of the form face wall adjacent to the compacted composite material; $L-$ the distance between the form face walls (the thickness of the compacted layer).

The first boundary condition (5) describes the interaction between the vertical plate and the compacted composite material and the second boundary condition (6) shows that the form face walls move synchronously. Based on function (4), expression $Q \sin \omega t$ in boundary condition (5) can be represented in the form of the imaginary part of the complex function, i.e. $Q \sin \omega t=Q e^{i \omega t}$.

We substitute expression (4) into equation (3) and find its solution in the following complex form:

$$
u(x, t)=e^{-\delta x}\left[B e^{(i x+\alpha) x}+D e^{-(i k+\alpha) x}\right] e^{i(\omega t-\xi x)} .
$$

where $B$ and $D$ - integration constants (complex functions), determined from boundary conditions (5) and (6); $k$-wave coefficient; $\alpha$ - absorption coefficient; $\delta$ - excitation attenuation coefficient; $\xi$ - dissipation coefficient;

$$
\begin{gathered}
k=\sqrt{\frac{1}{2}\left(\rho \omega^{2} / E+\xi^{2}-\delta^{2}\right)+\sqrt{\frac{1}{4}\left(\rho \omega^{2} / E+\xi^{2}-\delta^{2}\right)^{2}+\xi^{2} \delta^{2}}} ; \\
\alpha=\sqrt{-\frac{1}{2}\left(\rho \omega^{2} / E+\xi^{2}-\delta^{2}\right)+\sqrt{\frac{1}{4}\left(\rho \omega^{2} / E+\xi^{2}-\delta^{2}\right)^{2}+\xi^{2} \delta^{2}}} ; \\
\delta=\left(\mu+\rho L_{1} \omega^{2}\right) /(2 E) ; \quad \xi=\eta \omega /(2 E) .
\end{gathered}
$$

Substituting dependence (7) into boundary condition (6), we find the relation between integration constants $B$ and $D$ : 


$$
B=-D \frac{1-e^{-(\delta+\alpha) L} \cos (k+\xi) L+i \cdot e^{-(\delta+\alpha) L} \sin (k+\xi) L}{1-e^{-(\delta-\alpha) L} \cos (k-\xi) L-i \cdot e^{-(\delta-\alpha) L} \sin (k-\xi) L} .
$$

Substituting dependence (11) into expression (7), we find the solution to equation (3) in the following form:

$$
\begin{gathered}
u(x, t)=D e^{-\delta x}\left\{\frac{\left[1-e^{-(\delta-\alpha) L} \cos (k-\xi) L-i \cdot e^{-(\delta-\alpha) L} \sin (k-\xi) L\right] e^{-(\alpha+i k) x}}{1-e^{-(\delta-\alpha) L} \cos (k-\xi) L-i \cdot e^{-(\delta-\alpha) L} \sin (k-\xi) L}-\right. \\
\left.-\frac{\left[1-e^{-(\delta+\alpha) L} \cos (k+\xi) L+i \cdot e^{-(\delta+\alpha) L} \sin (k+\xi) L\right] e^{(\alpha+i k) x}}{1-e^{-(\delta-\alpha) L} \cos (k-\xi) L-i \cdot e^{-(\delta-\alpha) L} \sin (k-\xi) L}\right\} e^{i(\omega t-\xi x)} .
\end{gathered}
$$

To simplify further mathematical computations we transform the obtained expression (12), reducing it to the following form:

$$
u(x, t)=D e^{-\delta x}\left[\frac{(d-i \lambda) e^{-(\alpha+i k) x}-(M+i N) e^{(\alpha+i k) x}}{d-i \lambda}\right] e^{i(\omega t-\xi x)},
$$

where

$$
\begin{aligned}
& d=1-e^{-(\delta-\alpha) L} \cos (k-\xi) L ; \quad \lambda=e^{-(\delta-\alpha) L} \sin (k-\xi) L \\
& M=1-e^{-(\delta+\alpha) L} \cos (k+\xi) L ; \quad N=e^{-(\delta+\alpha) L} \sin (k+\xi) L
\end{aligned}
$$

By substituting dependence (13) into boundary condition (5) we find integration constant $D$ (a complex value):

$$
D=\frac{Q(d-i \lambda)}{(d-M)-i(\lambda+N)}\left[c-m \omega^{2}-0,5 F\left(\rho L_{1} \omega^{2}+\mu\right)-i \cdot 0,5 \eta \omega F+E F(\alpha+i k) \frac{(d+M)-i(\lambda-N)}{(d-M)-i(\lambda+N)}+\right]^{-1}
$$

Using expressions (14) - (15), we transform complex dependence $(d-M)+i(\lambda-N)$, in the denominator of expression (16), reducing it to the following form:

$$
(d-M)-i(\lambda+N)=R-i S,
$$

where $R$ and $S$ - the real and the imaginary parts of complex function (17),

$$
\begin{gathered}
R=d-M=-e^{-(\delta-\alpha) L} \cos (k-\xi) L+e^{-(\delta+\alpha) L} \cos (k+\xi) L \\
S=\lambda+N=e^{-(\delta-\alpha) L} \sin (k-\xi) L+e^{-(\delta+\alpha) L} \sin (k+\xi) L .
\end{gathered}
$$

We transform complex dependence $(d+M)-i(\lambda-N)$ in the analogous way and reduce it to the following form:

$$
(d+M)-i(\lambda-N)=R_{1}-i S_{1},
$$

where $R_{1}$ and $S_{1}$ - the real and the imaginary parts of complex function (19),

$$
\begin{gathered}
R_{1}=d+M=2-e^{-(\delta-\alpha) L} \cos (k-\xi) L-e^{-(\delta+\alpha) L} \cos (k+\xi) L \\
S_{1}=\lambda-N=e^{-(\delta-\alpha) L} \sin (k-\xi) L-e^{-(\delta+\alpha) L} \sin (k+\xi) L .
\end{gathered}
$$


Based on dependences (17) and (20), we reduce the expression for the determination of integration constant (16) to the following form:

$$
D=\frac{Q(d-i \lambda)}{\left.(R-i S)\left[c-m \omega^{2}-0,5 F\left(\rho L_{1} \omega^{2}+\mu\right)-i \cdot 0,5 \eta \omega F\right)+E F(\alpha+i k) \frac{R_{1}-i S_{1}}{R-i S}\right]} .
$$

Multiplying the numerator and denominator of expression $\frac{R_{1}-i S_{1}}{R-i S}$ in dependence (23) by a complex number conjugate with the denominator and, transforming this dependence taking into consideration expressions (18), (19), (21) and (22), we obtain

$$
D=\frac{Q(d-i \lambda)}{(R-i S)\left\{\left[c+c_{b}-\left(m+m_{b}\right) \omega^{2}\right]+i b_{b} \omega\right\}},
$$

where $c_{b}$ and $b_{b}$ - the reduced values of rigidity and the coefficient of non-resilient resistance of the compacted composite material; $m_{b}$ - the reduced mass of the compacted composite material,

$$
\begin{gathered}
c_{b}=\frac{2 E F}{R^{2}+S^{2}}\left\{k\left[e^{-(\delta+\alpha) L} \sin (k+\xi) L+e^{-(\delta-\alpha) L} \sin (k-\xi) L-e^{-2 \delta L} \sin 2 k L\right]-\right. \\
\left.-\alpha\left[e^{-(\delta+\alpha) L} \cos (k+\xi) L+e^{-(\delta-\alpha) L} \cos (k-\xi) L-e^{-2 \delta L} \operatorname{sh}(2 \alpha L)\right]\right\} ; \\
b_{b}=\frac{2 E F}{R^{2}+S^{2}}\left\{\alpha\left[e^{-(\delta+\alpha) L} \sin (k+\xi) L+e^{-(\delta-\alpha) L} \sin (k-\xi) L-e^{-2 \delta L} \sin 2 k L\right]+\right. \\
\left.+k\left[e^{-(\delta+\alpha) L} \cos (k+\xi) L+e^{-(\delta-\alpha) L} \cos (k-\xi) L-e^{-2 \delta L} \operatorname{sh}(2 \alpha L)\right]\right\}-0,5 \eta F . \\
m_{b}=F\left(\mu / \omega^{2}+0,5 \rho L_{1}\right) ;
\end{gathered}
$$

Substituting integration constant $D$ (24) into dependence (13), we find the solution to the oscillation wave equation (3), meeting boundary conditions (5) and (6), in a complex form:

$$
u(x, t)=Q e^{-\delta x} \frac{(d-i \lambda) e^{-(\alpha+i k) x}-(M+i N) e^{(\alpha+i k) x}}{(R-i S)\left\{\left[c_{b}-\left(m+m_{b}\right) \omega^{2}\right]+i b_{b} \omega\right\}} e^{i(\omega t-\xi x)} .
$$

We multiply the numerator and denominator of expression (28) by complex numbers $\left[c+c_{b}-\left(m+m_{b}\right) \omega^{2}\right]-i b_{b} \omega$ и $R+i S$, conjugate with complex numbers in the denominator and, singling the imaginary part of the formed complex function out of the obtained dependence and transforming it, we obtain the desired solution of the wave equation (3), meeting boundary conditions (5) and (6), in the following form:

$$
\begin{gathered}
u(x, t)=\frac{Q e^{-\delta x}}{\sqrt{R^{2}+S^{2}} \sqrt{\left[c_{b}-\left(m+m_{b}\right) \omega^{2}\right]^{2}+b_{b}^{2} \omega^{2}}} \times \\
\times\left\{\left[e^{-\alpha x}(d \cos k x-\lambda \sin k x)-e^{\alpha x}(M \cos -N \sin k x)\right] \sin (\omega t-\xi x-\varphi)-\right.
\end{gathered}
$$




$$
\left.-\left[e^{-\alpha x}(\lambda \cos k x+d \sin k x)+e^{\alpha x}(N \cos k x+M \sin k x)\right] \cos (\omega t-\xi x-\varphi)\right\},
$$

where $\varphi-$ the shift of phases between the amplitude of the exciting force and motion,

$$
\begin{gathered}
\varphi=\varphi_{1}-\varphi_{2} ; \\
\varphi_{1}=\operatorname{arctg} \frac{b_{b} \omega}{c+c_{b}-\left(m+m_{b}\right) \omega^{2}} ; \quad \varphi_{2}=\operatorname{arctg} \frac{S}{R} .
\end{gathered}
$$

The obtained solution (2) to the oscillation wave equation (3), meeting boundary conditions (5) and (6), describes the law of oscillations of the composite material compacted layer of the analyzed "vibration machine - composite medium" dynamic system depending on coordinate $x$, i.e. at $L \leq x \geq 0$. At $x=0$ this dependence describes the law of oscillations of the mixture layer adjacent to the form end face and simultaneously the law of oscillations of the end face, i.e.

$$
u(0, t)=\frac{Q[(d-M) \sin (\omega t-\varphi)-(\lambda+N) \cos (\omega t-\varphi)]}{\sqrt{R^{2}+S^{2}} \sqrt{\left[c+c_{b}-\left(m+m_{b}\right) \omega^{2}\right]^{2}+b_{b}^{2} \omega^{2}}}=A \sin \left(\omega t-\varphi_{1}\right),
$$

where $A-$ the amplitude of oscillations of the form end face walls,

$$
A=\frac{Q}{\sqrt{\left[c+c_{b}-\left(m+m_{b}\right) \omega^{2}\right]^{2}+b_{b}^{2} \omega^{2}}} .
$$

Substituting dependence (29), describing the law of oscillations of the analyzed dynamic system into equation (1), we determine the change of stresses occurring in the compacted layer under the action of the vibration excitation $Q \sin \omega t$ :

$$
\begin{gathered}
\sigma(x, t)=\frac{0,5 A e^{-\delta x} \sqrt{\left(\mu+\rho L_{1} \omega^{2}\right)^{2}+(\eta \omega)^{2}}}{\sqrt{R^{2}+S^{2}}}\left\{\left[\left(d e^{-\alpha x}-M e^{\alpha x}\right) \cos k x-\left(\lambda e^{-\alpha x}-N e^{\alpha x}\right) \sin k x\right] \times\right. \\
\left.\times \sin \left(\omega t-\xi x-\varphi+\varphi_{3}\right)-\left[\left(\lambda e^{-\alpha x}+N e^{\alpha x}\right) \cos k x+\left(d e^{-\alpha x}+M e^{\alpha x}\right) \sin k x\right] \cos \left(\omega t-\xi x-\varphi+\varphi_{3}\right)\right\}- \\
-\frac{A E e^{-\delta x} \sqrt{\alpha^{2}+k^{2}}}{\sqrt{\left.R^{2}+S^{2}\right)}}\left\{\left[\left(d e^{-\alpha x}+M e^{\alpha x}\right) \cos k x-\left(\lambda e^{-\alpha x}+N e^{\alpha x}\right) \sin k x\right] \cos \left(\omega t-\xi x-\varphi-\varphi_{4}\right)+\right. \\
\left.+\left[\left(\lambda e^{-\alpha x}-N e^{\alpha x}\right) \cos k x+\left(d e^{-\alpha x}-M e^{\alpha x}\right) \sin k x\right] \sin \left(\omega t-\xi x-\varphi-\varphi_{4}\right)\right\},
\end{gathered}
$$

where $\varphi_{3}, \varphi_{4}-$ phases shift angles,

$$
\varphi_{3}=\operatorname{arctg}\left[\eta \omega /\left(\mu+\rho L_{1} \omega^{2}\right)\right] ; \quad \varphi_{4}=\operatorname{arctg}(\alpha / k) .
$$

The stresses occurring in the composite medium where it contacts the form end face walls equal to: 


$$
\begin{aligned}
& \sigma(0, t)=\frac{0,5 A \sqrt{\left(\mu+\rho L_{1} \omega^{2}\right)^{2}+(\eta \omega)^{2}}}{\sqrt{R^{2}+S^{2}}}\left[(d-M) \sin \left(\omega t-\varphi+\varphi_{3}\right)-(\lambda+N) \cos \left(\omega t-\varphi+\varphi_{3}\right)\right]- \\
& -\frac{A E \sqrt{\alpha^{2}+k^{2}}}{\sqrt{\left.R^{2}+S^{2}\right)}}\left[(d+M) \cos \left(\omega t-\varphi-\varphi_{4}\right)+(\lambda-N) \sin \left(\omega t-\varphi-\varphi_{4}\right)\right] .
\end{aligned}
$$

To provide the efficient compaction of composite materials it is necessary to meet the condition when the stresses occurring in the compacted layer are to exceed the minimal values of the stresses needed for ultimate destruction of the structural links in the compacted medium, i.e.

$$
\sigma(x) \geq \sigma_{0 i}
$$

where $\sigma(x)$ - the amplitude of the stresses occurring in the compacted layer depending on coordinate $x ; \sigma_{0 i}$ - the value of the minimal stresses providing the ultimate destruction of the structural links in the compacted layer.

It is during the destruction of the structural links in the compacted layer that its internal friction coefficient sharply decreases, air is removed out of it, the mobility of the mixture components and their reorientation increases and a more compact packing is formed.

\section{Conclusion}

The authors have analyzed the process of deformation wave propagation in the compacted medium represented in the form of a system with distributed parameters and have determined the law of deformation of the compacted medium when it undergoes oscillations horizontally directed to it from the form vertical walls. The authors have obtained theoretical expressions making it possible to describe with sufficient accuracy the behavior of the "vibration machine - compacted medium" real dynamic system during the formation of composite products by application of horizontally directed forces. They enable computer modeling of the laws of motion and forms of oscillations of the composite material compacted layers, their analysis from the point of view of the horizontally directed oscillation effective action on the processed medium, the substantiation of the type and form of the vibratory action and the substantiation of the rational parameters of vibration equipment.

\section{References}

1. Y.V. Holodnikov, Methods of manufacturing products from composites, I. J. Appl. and Fundamental Research, v.6, pp. 214 - 221 (2016)

2. N.A. Shestakov, V.N. Subich, V.A. Demin, Compaction, consolidation and destruction of porous materials (M., Fizmatlit, 2011)

3. J. Gutierrez, E. Ruiz and F.Trochu, High-frequency vibrations on the compaction of dry fibrous reinforcements, Advanced Composite Materials, v. 22 (1), (2013)

4. X. Chen, S. Wu and J. Zhou, Experimental study and analytical formulation of mechanical behavior of concrete, Construction and Buildings Materials, v. 47, pp. 662-670 (2013). 
5. P. F. G. Banfill, M. A. O. M. Teixeira and R. J. M.Craik, Rheology and vibration of fresh concrete: Predicting the radius of action of poker vibrators from wave propagation, Cement and Concert Research, v. 41(9), pp. 932-941 (2011)

6. C.A. Braun, M. Schumaker, J. Rice and J.P. Borg, Comparison of Static and Dynamic Powder Compaction: Experiment and Simulation, J. Eng. Mater. Technol, 138(1), 011003 (2015)

7. H. Abdellaoui and J. Echaabi, Rheological models for modeling the viscoelastic behavior in liquid composite molding processes (LCM) review, J. Reinforced Plastics and Composites, 33, 8, (714), (2014)

8. H. Sepani, M.A. Polak and A. Penlidis, Constitutive Equations and Finite Element Implementation of Isochronous Nonlinear Viscoelastic Behavior, J. Eng. Mater. Technol, 140(4), 041004 (2018)

9. A.G. Maslov and Y.S. Salenko, Vibrating machines and processes in road construction industry: monograph (PB Cherbatyh, Kremenchuk, 2014) 